\title{
A Need for Logical and Consistent Anatomical Nomenclature for Cutaneous Nerves of the Limbs
}

\author{
Thomas R. Gest, ${ }^{*}$ William E. Burkel, Gerald W. Cortright \\ Division of Anatomical Sciences, University of Michigan Medical School, Ann Arbor, Michigan
}

\begin{abstract}
The system of anatomical nomenclature needs to be logical and consistent. However, variations in translation to English of the Latin and Greek terminology used in Nomina Anatomica and Terminologia Anatomica have led to some inconsistency in the nomenclature of cutaneous nerves in the limbs. An historical review of cutaneous nerve nomenclature reveals that there are two general naming conventions: one primarily American and one primarily British. The American convention presents cutaneous nerves of the limbs in the format "medial brachial cutaneous nerve," while the British convention presents the same nerve as "medial cutaneous nerve of the arm," thereby translating "brachii" to "of the arm." If logically and consistently applied throughout the body, the British convention would rename the sural nerve to the "nerve of the calf," the brachial artery would become the "artery of the arm," the femoral nerve would be "nerve of the thigh," and femur would be "bone of the thigh" or "thigh bone." The British convention leads to many other nomenclatural inconsistencies, which would seem to make learning anatomy more difficult for the beginning student. In this era of contracting anatomy curricula, every effort should be made to keep anatomical nomenclature simple, logical, and consistent. Anat Sci Ed 2:126-134, 2009. @ 2009 American Association of Anatomists.
\end{abstract}

Key words: cutaneous nerves; anatomical terminology; anatomical nomenclature; Terminologia Anatomica; FCAT

\section{INTRODUCTION}

Anatomy teachers know that the primary task that confronts the student of gross anatomy is the acquisition of a new vocabulary of human body structure. At one time, prior to the establishment of international nomenclatural rules, anatomical terminology as presented in textbooks contained a morass of descriptive terms and eponyms. Colorful terms, such as the musculospiral nerve for the radial nerve (Gray, 1858) and Poupart's ligament for the inguinal ligament (Gray, 1858), may have been memorable, but without international standards, nomenclature varied considerably from text to text. With the establishment of the Basle Nomina Anatomica in 1895 (His, 1895), anatomy nomenclature was placed on a path of regulation and standardization. There have been

*Correspondence to: Dr. Thomas R. Gest, Division of Anatomical Sciences, University of Michigan Medical School, Ann Arbor, MI 48109-0608, USA. E-mail: gest@umich.edu

Received 22 April 2009; Revised 8 May 2009; Accepted 11 May 2009. Published online 8 June 2009 in Wiley InterScience (www. interscience.wiley.com). DOI 10.1002/ase.90

(C) 2009 American Association of Anatomists numerous adjustments to the system of anatomical terminology, culminating in 1998 with the publication of Terminologia Anatomica or TA (FCAT, 1998). In this guide, Latin/ Greek terms are accompanied by English equivalents. However, it is the English equivalent that presents a problem. In the preface to TA, it is stated that Latin terms would be "accompanied by a term in current usage in English-speaking countries" (FCAT, 1998). The problem arises as to which terms are considered to be in current usage in English-speaking countries.

Unfortunately, the Latin anatomical terminology in TA can be translated in various ways, and the resulting variation in terminology can, and almost certainly does, cause confusion for the beginning student of anatomy. Most of the variations in translation occur in the terminology for the cutaneous nerves of the limbs. As an example, TA offers two acceptable English equivalents for "Nervus cutaneous brachii medialis": "medial brachial cutaneous nerve" and "medial cutaneous nerve of the arm." To the seasoned anatomist, these are obviously alternate translations. However, to the beginning student of anatomy, the relationship between these terms may not be clear at all. To investigate the source of this potential confusion, we have undertaken an historical review of the terminology used for cutaneous nerves of the limbs. In the hope of saving future students from unnecessary 
sources of confusion, we propose a modification to our system of nomenclature that employs logic and consistency.

\section{MATERIALS AND METHODS}

One hundred and fifty-two anatomy books and atlases in the holdings of the authors and the University of Michigan Medical Library were reviewed to discern the type of terminology used for the cutaneous nerves of the limbs. These extensive holdings, while not comprehensive, represent a reasonable sampling of anatomy textbooks and atlases used during the past century and a half. The year of publication, affiliation(s) of the author(s), and classification of the terminology were recorded. Five categories of terminology were determined. Category 1 was termed "unclassified," and these books did not follow TA or Nomina Anatomica (IANC, 1983). Category 2 was termed "untranslated," and these books retained the original Latin terminology. Category 3 was termed "American," and these books used the format "medial brachial cutaneous nerve." Category 4 was termed "British," and these books used the format "medial cutaneous nerve of the arm." Category 5 was termed "both," and these books used both American and British systems at various locations within the limbs. As seen in the results, Category 3 was termed "American" because the majority of authors using this terminology had affiliations with U.S. schools. Similarly, Category 4 was termed "British" because authors in this category were most commonly affiliated with British schools. The relationship between terminology category and affiliation is treated in greater depth in the results section.

\section{RESULTS}

Table 1 shows the classification data of the anatomy texts used in the present study. Five books were placed in Category 1, the "unclassified" category. Three of these (Bell, 1834; Gray, 1858; Wilson, 1858) predated the Basle Nomina Anatomica (His, 1895), while the most recent "unclassified" text was on surgical anatomy (Piersol, 1923). Nine books were in Category 2, "untranslated," meaning that Latin terminology was used, and most of these texts were English editions of German texts (i.e., Anson, 1950; Anson and Maddock, 1952; Pernkopf, 1964, 1980; Spalteholz, 1900; Spalteholz and Spanner, 1967). In Category 5, 13 books used "both" the American and British nomenclature in various locations. (DiDio, 1970; Tobin, 1973; McMinn et al., 1984; Gosling et al., 1985; Martin, 1985; Callas, 1994; Ger et al., 1996; Backhouse and Hutchings, 1998; Putz and Pabst, 2000; Lumley et al., 1995, 2002; Rohen et al., 2002; Abrahams et al., 2003). For most of these, the British system was used in the upper limb while the American system was used in the lower limb. For several books, the "lateral femoral cutaneous nerve" was the only exception to an otherwise British rule. One of the "both" books was atypical in presenting the British nomenclature with the American nomenclature in parentheses.

Seventy-five books were placed in Category 3, using the American nomenclature system, while 50 books were placed in Category 4, the British nomenclature system (refer to Table 1). The first book to use the American nomenclature appeared in 1906 (Sobotta and McMurrich, 1906) while the first book to use the British nomenclature appeared in 1937 (Pauchet and Dupret, 1937). The historical data are summar- ized in Figure 1. It is not at all surprising that all but 12 of the authors of American nomenclature books had affiliations with American schools, but it is somewhat surprising that none of the 12 with non-American affiliations were British. On the other hand, 16 of the books using the British nomenclature system had authors with American affiliations, and 28 were not affiliated with British schools. Of the 16 books using the British nomenclature system by authors with American affiliations, 7 were published within the past decade, and 5 within the past 4 years. The first text by an American author using the British nomenclature system appeared in 1959 (Francis, 1959). Unfortunately, it was not always possible to discern the place of training of authors, and undoubtedly this may have influenced the choice of nomenclature systems. Of the 12 authors using the British nomenclature system with affiliations at schools other than British or American, there were eight authors with affiliations in Canada, and one each from France, Australia, India, and the Netherlands.

\section{DISCUSSION}

As teachers of anatomy, we strive to make our subject accessible for our students. The most difficult task in learning anatomy is mastering the huge new vocabulary of the human body. It should be our goal, then, to present anatomical terminology in the most comprehensible manner possible. We suggest that a logical and consistent translation of the Latin that serves as the foundation of anatomical terminology is our duty as anatomy educators. Confusion results when the Latin terminology is not translated consistently and logically. In the British nomenclature system, Latin translation occurs in one manner for cutaneous nerves, and in another manner for other structures. Hence, in the British system, "arteria femoralis" is "femoral artery," and "nervus femoralis" is "femoral nerve," but "nervus cutaneus femoris lateralis" is "lateral cutaneous nerve of the thigh."

The conflict of logical and consistent translations is particularly evident where the limbs meet the trunk. Here, in the upper limb, we find the intercostobrachial nerve communicating with the medial brachial cutaneous nerve or, if the British system is logically applied, the medial cutaneous nerve of the arm communicates with the intercostal nerve of the arm. In the American system, the anterior thigh is innervated by the lateral femoral cutaneous nerve, anterior femoral cutaneous branches of the femoral nerve, and the femoral branch of the genitofemoral nerve, whereas in a consistent application of the British system, this region is supplied by the lateral cutaneous nerve of the thigh, anterior cutaneous branches of the thigh nerve, and the thigh branch of the genital thigh nerve.

The Terminologia Anatomica itself exhibits inconsistency in translation. As an example, the "os femoris" is translated as "thigh bone," while "corpus femoris" is "body of femur" and "caput femoris" is "head of femur." If translation to English equivalents were consistent, "corpus femoris" would be translated to "thigh body" and "caput femoris" would be "thigh head." As noted earlier, "n. cutaneus femoris lateralis" is translated in TA to "lateral cutaneous nerve of the thigh," while " $n$. cutaneus surae lateralis" is translated to "lateral sural cutaneous nerve" and "n. suralis" is "sural nerve." For the nomenclature system to be consistent and logical, this should be "lateral cutaneous nerve of the calf," and "n. suralis" should be "calf nerve." 
Table 1.

Anatomy Texts Classified by Nomenclature System

\begin{tabular}{|c|c|c|}
\hline Author(s) & $\begin{array}{c}\text { Publication } \\
\text { date }\end{array}$ & Title \\
\hline \multicolumn{3}{|c|}{ Category 1: Unclassified nomenclature } \\
\hline Bell & 1834 & $\begin{array}{l}\text { The Anatomy and } \\
\text { Physiology of } \\
\text { the Human Body, } \\
\text { Vol.1 }\end{array}$ \\
\hline Gray & 1858 & $\begin{array}{l}\text { Anatomy, Descriptive } \\
\text { and Surgical }\end{array}$ \\
\hline $\begin{array}{l}\text { Pick and } \\
\text { Howden }\end{array}$ & 1901 & $\begin{array}{l}\text { Anatomy, Descriptive } \\
\text { and Surgical }\end{array}$ \\
\hline Piersol & 1923 & Human Anatomy \\
\hline Wilson & 1858 & $\begin{array}{l}\text { A System of Human } \\
\text { Anatomy }\end{array}$ \\
\hline \multicolumn{3}{|c|}{ Category 2: Untranslated nomenclature } \\
\hline Anson & 1950 & $\begin{array}{l}\text { An Atlas of Human } \\
\text { Anatomy }\end{array}$ \\
\hline $\begin{array}{l}\text { Anson and } \\
\text { Maddock }\end{array}$ & 1952 & $\begin{array}{l}\text { Callander's Surgical } \\
\text { Anatomy }\end{array}$ \\
\hline Callander & 1933 & Surgical Anatomy \\
\hline Callander & 1939 & Surgical Anatomy \\
\hline Pernkopf & 1964 & $\begin{array}{l}\text { Atlas of Topographical } \\
\text { and Applied Human } \\
\text { Anatomy }\end{array}$ \\
\hline Pernkopf & 1980 & $\begin{array}{l}\text { Atlas of Topographical } \\
\text { and Applied Human } \\
\text { Anatomy }\end{array}$ \\
\hline Spalteholz & 1900 & $\begin{array}{l}\text { Hand-Atlas of Human } \\
\text { Anatomy }\end{array}$ \\
\hline $\begin{array}{l}\text { Spalteholz } \\
\text { and Spanner }\end{array}$ & 1967 & $\begin{array}{l}\text { Atlas of Human } \\
\text { Anatomy }\end{array}$ \\
\hline Wolf-Heidegger & 1962 & $\begin{array}{c}\text { Atlas of Systematic } \\
\text { Human Anatomy }\end{array}$ \\
\hline \multicolumn{3}{|c|}{ Category 3: American nomenclature system } \\
\hline Agur & 1991 & $\begin{array}{l}\text { Grant's Atlas of } \\
\text { Anatomy }\end{array}$ \\
\hline $\begin{array}{l}\text { Agur and } \\
\text { Dalley }\end{array}$ & 2005 & $\begin{array}{l}\text { Grant's Atlas of } \\
\text { Anatomy }\end{array}$ \\
\hline Anson & 1966 & $\begin{array}{l}\text { Morris' Human } \\
\text { Anatomy }\end{array}$ \\
\hline Arnold & 1968 & $\begin{array}{l}\text { Reconstructive } \\
\text { Anatomy }\end{array}$ \\
\hline
\end{tabular}

Table 1.

(Continued)

\begin{tabular}{|c|c|c|}
\hline Author(s) & $\begin{array}{l}\text { Publication } \\
\text { date }\end{array}$ & Title \\
\hline Beck & 1982 & $\begin{array}{l}\text { Mosby's Atlas of } \\
\text { Functional } \\
\text { Human Anatomy }\end{array}$ \\
\hline Brantigan & 1963 & Clinical Anatomy \\
\hline $\begin{array}{l}\text { Christensen } \\
\text { and Telford }\end{array}$ & 1966 & $\begin{array}{l}\text { Synopsis of Gross } \\
\text { Anatomy }\end{array}$ \\
\hline Chung & 1991 & Gross Anatomy \\
\hline Clemente & 1975 & $\begin{array}{l}\text { Anatomy: A Regional } \\
\text { Atlas of the Human } \\
\text { Body }\end{array}$ \\
\hline Clemente & 1985 & Gray's Anatomy \\
\hline Clemente & 1997 & $\begin{array}{l}\text { Anatomy: A Regional } \\
\text { Atlas of the Human } \\
\text { Body }\end{array}$ \\
\hline $\begin{array}{l}\text { Colborn and } \\
\text { Skandalakis }\end{array}$ & 1993 & Clinical Gross Anatomy \\
\hline Crafts & 1966 & $\begin{array}{l}\text { A Textbook of Human } \\
\text { Anatomy }\end{array}$ \\
\hline Crafts & 1985 & $\begin{array}{l}\text { A Textbook of Human } \\
\text { Anatomy }\end{array}$ \\
\hline Crouch & 1972 & $\begin{array}{l}\text { Functional Human } \\
\text { Anatomy }\end{array}$ \\
\hline Dawson & 1996 & Basic Human Anatomy \\
\hline Frick et al. & 1991 & Human Anatomy \\
\hline $\begin{array}{l}\text { Gardner and } \\
\text { Osburn }\end{array}$ & 1973 & $\begin{array}{l}\text { Structure of the Human } \\
\text { Body }\end{array}$ \\
\hline $\begin{array}{l}\text { Gardner and } \\
\text { Osburn }\end{array}$ & 1978 & $\begin{array}{l}\text { Anatomy of the Human } \\
\text { Body }\end{array}$ \\
\hline $\begin{array}{l}\text { Gest and } \\
\text { Schlesinger }\end{array}$ & 1994 & Medcharts: Anatomy \\
\hline Goss & 1954 & $\begin{array}{l}\text { Gray's Anatomy of the } \\
\text { Human Body }\end{array}$ \\
\hline Goss & 1959 & $\begin{array}{l}\text { Gray's Anatomy of the } \\
\text { Human Body }\end{array}$ \\
\hline Goss & 1966 & $\begin{array}{l}\text { Gray's Anatomy of the } \\
\text { Human Body }\end{array}$ \\
\hline Gottlieb & 1964 & Anatomy Review \\
\hline Gray & 1948 & $\begin{array}{l}\text { Anatomy of the Human } \\
\text { Body }\end{array}$ \\
\hline Hall-Craggs & 1990 & $\begin{array}{l}\text { Anatomy as a Basis for } \\
\text { Clinical Medicine }\end{array}$ \\
\hline
\end{tabular}


Table 1.

(Continued)

\begin{tabular}{|c|c|c|}
\hline Author(s) & $\begin{array}{c}\text { Publication } \\
\text { date }\end{array}$ & Title \\
\hline Hansen & 1998 & $\begin{array}{l}\text { Essential Anatomy } \\
\text { Dissector }\end{array}$ \\
\hline Healey & 1969 & $\begin{array}{l}\text { A Synopsis of } \\
\text { Clinical Anatomy }\end{array}$ \\
\hline Hollinshead & 1969 & $\begin{array}{l}\text { Anatomy for } \\
\text { Surgeons }\end{array}$ \\
\hline Hollinshead & 1974 & $\begin{array}{l}\text { Textbook of } \\
\text { Anatomy }\end{array}$ \\
\hline $\begin{array}{l}\text { Hollinshead } \\
\text { and Rosse }\end{array}$ & 1985 & $\begin{array}{l}\text { Textbook of } \\
\text { Anatomy }\end{array}$ \\
\hline Huber & 1930 & $\begin{array}{l}\text { Piersol's Human } \\
\text { Anatomy }\end{array}$ \\
\hline Jackson & 1914 & $\begin{array}{l}\text { Morris's Human } \\
\text { Anatomy }\end{array}$ \\
\hline Jacobs & 1989 & $\begin{array}{l}\text { Shearer's Manual of } \\
\text { Human Dissection }\end{array}$ \\
\hline Kahle et al. & 1992 & $\begin{array}{l}\text { Color Atlas and } \\
\text { Textbook of } \\
\text { Human Anatomy. } \\
\text { Vol. } 1 \text { Locomotor } \\
\text { System }\end{array}$ \\
\hline $\begin{array}{l}\text { Kanagasuntheram } \\
\text { et al. }\end{array}$ & 1987 & $\begin{array}{l}\text { Anatomy: Regional, } \\
\text { Functional, and } \\
\text { Clinical }\end{array}$ \\
\hline Langebartel & 1977 & $\begin{array}{l}\text { The Anatomical } \\
\text { Primer: An } \\
\text { Embryological } \\
\text { Explanation of } \\
\text { Human Gross } \\
\text { Morphology }\end{array}$ \\
\hline $\begin{array}{l}\text { Langman and } \\
\text { Woerdeman }\end{array}$ & 1978 & $\begin{array}{l}\text { Atlas of Medical } \\
\text { Anatomy }\end{array}$ \\
\hline $\begin{array}{l}\text { Leeson and } \\
\text { Leeson }\end{array}$ & 1972 & $\begin{array}{l}\text { Human Structure: A } \\
\text { Companion to } \\
\text { Anatomical } \\
\text { Studies }\end{array}$ \\
\hline Leonard & 1995 & $\begin{array}{l}\text { Human Gross } \\
\text { Anatomy }\end{array}$ \\
\hline Lewis & 1918 & $\begin{array}{l}\text { Gray's Anatomy of } \\
\text { the Human Body }\end{array}$ \\
\hline Lewis & 1936 & $\begin{array}{l}\text { Gray's Anatomy of } \\
\text { the Human Body }\end{array}$ \\
\hline Lewis & 1942 & $\begin{array}{l}\text { Gray's Anatomy of } \\
\text { the Human Body }\end{array}$ \\
\hline
\end{tabular}

Table 1.

(Continued)

\begin{tabular}{|c|c|c|}
\hline Author(s) & $\begin{array}{c}\text { Publication } \\
\text { date }\end{array}$ & Title \\
\hline $\begin{array}{l}\text { Lopez-Antunez } \\
\text { and Gasparo }\end{array}$ & 1971 & $\begin{array}{l}\text { Atlas of Human } \\
\text { Anatomy }\end{array}$ \\
\hline Mizeres & 1981 & $\begin{array}{l}\text { Human Anatomy: A } \\
\text { Synoptic } \\
\text { Approach }\end{array}$ \\
\hline Moore & 1992 & $\begin{array}{l}\text { Clinically Oriented } \\
\text { Anatomy }\end{array}$ \\
\hline Netter & 1987 & $\begin{array}{l}\text { The Ciba Collection } \\
\text { of Medical } \\
\text { Illustrations }\end{array}$ \\
\hline Netter & 1989 & $\begin{array}{l}\text { Atlas of Human } \\
\text { Anatomy }\end{array}$ \\
\hline Netter & 2006 & $\begin{array}{l}\text { Atlas of Human } \\
\text { Anatomy }\end{array}$ \\
\hline $\begin{array}{l}\text { Olson and } \\
\text { Pawlina }\end{array}$ & 1996 & $\begin{array}{l}\text { A.D.A.M. Student } \\
\text { Atlas of Anatomy }\end{array}$ \\
\hline $\begin{array}{l}\text { O'Rahilly and } \\
\text { Müeller }\end{array}$ & 1983 & $\begin{array}{l}\text { Basic Human } \\
\text { Anatomy: A } \\
\text { Regional Study of } \\
\text { Human Structure }\end{array}$ \\
\hline O'Rahilly & 1986 & $\begin{array}{l}\text { Anatomy: A } \\
\text { Regional Study } \\
\text { of Human } \\
\text { Structure }\end{array}$ \\
\hline $\begin{array}{l}\text { Pansky and } \\
\text { House }\end{array}$ & 1975 & $\begin{array}{l}\text { Review of Gross } \\
\text { Anatomy }\end{array}$ \\
\hline Philo et al. & 1985 & $\begin{array}{l}\text { Guide to Human } \\
\text { Anatomy }\end{array}$ \\
\hline $\begin{array}{l}\text { Quiring and } \\
\text { Warfel }\end{array}$ & 1960 & The Extremities \\
\hline Rarey et al. & 1994 & $\begin{array}{l}\text { Human Anatomy: } \\
\text { Manual of Human } \\
\text { Dissection }\end{array}$ \\
\hline $\begin{array}{l}\text { Rosse and } \\
\text { Gaddum- } \\
\text { Rosse }\end{array}$ & 1997 & $\begin{array}{l}\text { Hollinshead's } \\
\text { Textbook of } \\
\text { Anatomy }\end{array}$ \\
\hline Schaeffer & 1942 & $\begin{array}{l}\text { Morris' Human } \\
\text { Anatomy }\end{array}$ \\
\hline Slaby et al. & 1994 & $\begin{array}{l}\text { Gross Anatomy in } \\
\text { the Practice of } \\
\text { Medicine }\end{array}$ \\
\hline $\begin{array}{l}\text { Sobotta and } \\
\text { McMurrich }\end{array}$ & 1906 & $\begin{array}{l}\text { Atlas and Text-Book } \\
\text { of Human } \\
\text { Anatomy }\end{array}$ \\
\hline
\end{tabular}

(Continued) 
Table 1.

(Continued)

\begin{tabular}{|c|c|c|}
\hline Author(s) & $\begin{array}{c}\text { Publication } \\
\text { date }\end{array}$ & Title \\
\hline $\begin{array}{l}\text { Sobotta and } \\
\text { McMurrich }\end{array}$ & 1928 & $\begin{array}{l}\text { Atlas of Human } \\
\text { Anatomy }\end{array}$ \\
\hline $\begin{array}{l}\text { Sobotta and } \\
\text { McMurrich }\end{array}$ & 1936 & Atlas of Human Anatomy \\
\hline Spence & 1986 & Basic Human Anatomy \\
\hline Stern & 1988 & $\begin{array}{l}\text { Essentials of Gross } \\
\text { Anatomy }\end{array}$ \\
\hline Tank & 2008 & Grant's Dissector \\
\hline $\begin{array}{l}\text { Tank and } \\
\text { Gest }\end{array}$ & 2008 & $\begin{array}{l}\text { Lippincott Williams and } \\
\text { Wilkins Atlas of } \\
\text { Anatomy }\end{array}$ \\
\hline Thiel & 1997 & $\begin{array}{c}\text { Photographic Atlas of } \\
\text { Practical Anatomy }\end{array}$ \\
\hline $\begin{array}{l}\text { Van De } \\
\text { Graaff }\end{array}$ & 2002 & Human Anatomy \\
\hline Vidić & 1984 & Manual of Dissection \\
\hline Vidić & 1984 & $\begin{array}{l}\text { Photographic Atlas } \\
\text { of the Human Body }\end{array}$ \\
\hline $\begin{array}{l}\text { Wilson and } \\
\text { Wilson }\end{array}$ & 1978 & Human Anatomy \\
\hline Wilson & 1988 & Dissection Manual \\
\hline Wischnitzer & 1972 & $\begin{array}{l}\text { Outline of Human } \\
\text { Anatomy }\end{array}$ \\
\hline $\begin{array}{l}\text { Woodburne } \\
\text { and } \\
\text { Burkel }\end{array}$ & 1994 & $\begin{array}{l}\text { Essentials of Human } \\
\text { Anatomy }\end{array}$ \\
\hline Zuidema & 1980 & $\begin{array}{c}\text { The Johns Hopkins Atlas } \\
\text { of Human Anatomy }\end{array}$ \\
\hline \multicolumn{3}{|c|}{ Category 4: British nomenclature system } \\
\hline Aitken et al. & 1956 & $\begin{array}{l}\text { A Manual of Human } \\
\text { Anatomy III }\end{array}$ \\
\hline $\begin{array}{l}\text { Akesson } \\
\text { et al. }\end{array}$ & 1990 & $\begin{array}{l}\text { Thompson's Core } \\
\text { Textbook of Anatomy }\end{array}$ \\
\hline Anderson & 1978 & $\begin{array}{l}\text { Grant's Atlas of } \\
\text { Anatomy }\end{array}$ \\
\hline Anderson & 1983 & $\begin{array}{l}\text { Grant's Atlas of } \\
\text { Anatomy }\end{array}$ \\
\hline Basmajian & 1976 & Primary Anatomy \\
\hline $\begin{array}{l}\text { Basmajian } \\
\text { and } \\
\text { Slonecker }\end{array}$ & 1989 & $\begin{array}{l}\text { Grant's Method of } \\
\text { Anatomy }\end{array}$ \\
\hline
\end{tabular}

Table 1.

(Continued)

\begin{tabular}{|c|c|c|}
\hline Author(s) & $\begin{array}{l}\text { Publication } \\
\text { date }\end{array}$ & Title \\
\hline Boyd et al. & 1956 & $\begin{array}{l}\text { Textbook of Human } \\
\text { Anatomy }\end{array}$ \\
\hline Brash & 1957 & $\begin{array}{l}\text { Cunningham's Manual of } \\
\text { Practical Anatomy }\end{array}$ \\
\hline $\begin{array}{l}\text { Cartmill } \\
\text { et al. }\end{array}$ & 1987 & Human Structure \\
\hline Drake et al. & 2005 & $\begin{array}{l}\text { Gray's Anatomy for } \\
\text { Students }\end{array}$ \\
\hline Ellis & 1960 & Clinical Anatomy \\
\hline Ellis & 1999 & $\begin{array}{l}\text { Human Sectional } \\
\text { Anatomy }\end{array}$ \\
\hline Ellis et al. & 1966 & Clinical Anatomy \\
\hline Francis & 1959 & $\begin{array}{l}\text { Introduction to Human } \\
\text { Anatomy }\end{array}$ \\
\hline $\begin{array}{l}\text { Gosling } \\
\text { et al. }\end{array}$ & 2002 & $\begin{array}{l}\text { Human Anatomy Color } \\
\text { Atlas and Text }\end{array}$ \\
\hline Grant & 1958 & A Method of Anatomy \\
\hline Grant & 1962 & An Atlas of Anatomy \\
\hline $\begin{array}{l}\text { Grant and } \\
\text { Basmajian }\end{array}$ & 1965 & $\begin{array}{l}\text { Grant's Method of } \\
\text { Anatomy }\end{array}$ \\
\hline $\begin{array}{l}\text { Green and } \\
\text { Silver }\end{array}$ & 1981 & $\begin{array}{l}\text { An Introduction to } \\
\text { Human } \\
\text { Anatomy }\end{array}$ \\
\hline Hamilton & 1976 & $\begin{array}{l}\text { Textbook of Human } \\
\text { Anatomy }\end{array}$ \\
\hline Jacob & 2002 & Atlas of Human Anatomy \\
\hline Jamieson & 1939 & $\begin{array}{l}\text { Illustrations of Regional } \\
\text { Anatomy }\end{array}$ \\
\hline Jamieson & 1950 & $\begin{array}{l}\text { A Companion to } \\
\text { Manuals of Practical } \\
\text { Anatomy }\end{array}$ \\
\hline Lachman & 1965 & $\begin{array}{l}\text { Case Studies in } \\
\text { Anatomy }\end{array}$ \\
\hline Last & 1955 & Aids to Anatomy \\
\hline $\begin{array}{l}\text { Lockhart } \\
\text { et al. }\end{array}$ & 1959 & $\begin{array}{l}\text { Anatomy of the Human } \\
\text { Body }\end{array}$ \\
\hline $\begin{array}{l}\text { McMinn and } \\
\text { Hutchings }\end{array}$ & 1977 & $\begin{array}{l}\text { Color Atlas of Human } \\
\text { Anatomy }\end{array}$ \\
\hline $\begin{array}{r}\text { McMinn and } \\
\text { Hutchings }\end{array}$ & 1988 & $\begin{array}{l}\text { Color Atlas of Human } \\
\text { Anatomy }\end{array}$ \\
\hline
\end{tabular}


Table 1.

(Continued)

\begin{tabular}{|c|c|c|}
\hline Author(s) & $\begin{array}{c}\text { Publication } \\
\text { date }\end{array}$ & Title \\
\hline $\begin{array}{l}\text { Melloni } \\
\text { et al. }\end{array}$ & 1988 & $\begin{array}{l}\text { Melloni's Illustrated } \\
\text { Review of Human } \\
\text { Anatomy }\end{array}$ \\
\hline $\begin{array}{l}\text { Mitchell } \\
\text { and } \\
\text { Patterson }\end{array}$ & 1967 & Basic Anatomy \\
\hline $\begin{array}{l}\text { Moore and } \\
\text { Dalley }\end{array}$ & 2006 & $\begin{array}{l}\text { Clinically Oriented } \\
\text { Anatomy }\end{array}$ \\
\hline $\begin{array}{l}\text { Morton } \\
\text { et al. }\end{array}$ & 2004 & $\begin{array}{l}\text { Dissection Guide for } \\
\text { Human Anatomy }\end{array}$ \\
\hline $\begin{array}{l}\text { Netter and } \\
\text { Dalley }\end{array}$ & 1997 & Atlas of Human Anatomy \\
\hline $\begin{array}{l}\text { Netter and } \\
\text { Hansen }\end{array}$ & 2003 & Atlas of Human Anatomy \\
\hline $\begin{array}{l}\text { Pauchet } \\
\text { and } \\
\text { Dupret }\end{array}$ & 1937 & Pocket Atlas of Anatomy \\
\hline Rogers & 1992 & Textbook of Anatomy \\
\hline Romanes & 1964 & $\begin{array}{l}\text { Cunningham's Textbook } \\
\text { of Anatomy }\end{array}$ \\
\hline Romanes & 1986 & $\begin{array}{l}\text { Cunningham's Manual of } \\
\text { Practical Anatomy, } \\
\text { Vol. 1: Upper and } \\
\text { Lower Limbs. }\end{array}$ \\
\hline Sahana & 1962 & Human Anatomy \\
\hline Sauerland & 1984 & Grant's Dissector \\
\hline Schade & 1970 & $\begin{array}{l}\text { Introduction to } \\
\text { Functional } \\
\text { Human Anatomy }\end{array}$ \\
\hline Sinclair & 1961 & $\begin{array}{l}\text { An Introduction to } \\
\text { Functional Anatomy }\end{array}$ \\
\hline Snell & 1978 & Atlas of Clinical Anatomy \\
\hline Snell & 1995 & $\begin{array}{l}\text { Clinical Anatomy for } \\
\text { Medical Students }\end{array}$ \\
\hline Srebnik & 2002 & Concepts in Anatomy \\
\hline
\end{tabular}

In some texts, a combination of American and British terminology styles is used (DiDio, 1970; Tobin, 1973; McMinn et al., 1984; Gosling et al., 1985; Martin, 1985; Callas, 1994; Ger et al., 1996; Backhouse and Hutchings, 1998; Putz and Pabst, 2000; Lumley et al., 1995, 2002; Rohen et al., 2002; Abrahams et al., 2003). This usually takes the form of the British style being used for the upper limb, and the American style being used for the lower limb. This would seem to aid in confusing the
Table 1.

(Continued)

\begin{tabular}{|c|c|c|}
\hline Author(s) & $\begin{array}{l}\text { Publication } \\
\text { date }\end{array}$ & Title \\
\hline $\begin{array}{l}\text { Standring } \\
\text { et al. }\end{array}$ & 2005 & Gray's Anatomy \\
\hline Thompson & 1977 & $\begin{array}{l}\text { Core Textbook of } \\
\text { Anatomy }\end{array}$ \\
\hline Thompson & 2002 & $\begin{array}{l}\text { Netter's Concise Atlas of } \\
\text { Orthopedic Anatomy }\end{array}$ \\
\hline Thorek & 1962 & Anatomy in Surgery \\
\hline Zuckerman & 1961 & $\begin{array}{l}\text { A New System of } \\
\text { Anatomy }\end{array}$ \\
\hline \multicolumn{3}{|c|}{ Category 5: Both nomenclature systems } \\
\hline $\begin{array}{l}\text { Abrahams } \\
\text { et al. }\end{array}$ & 2003 & $\begin{array}{l}\text { McMinn's Color Atlas } \\
\text { of Human Anatomy }\end{array}$ \\
\hline $\begin{array}{l}\text { Backhouse } \\
\text { and } \\
\text { Hutchings }\end{array}$ & 1998 & $\begin{array}{l}\text { Clinical Surface } \\
\text { Anatomy }\end{array}$ \\
\hline Callas & 1994 & $\begin{array}{l}\text { Manual for Human } \\
\text { Dissection }\end{array}$ \\
\hline DiDio & 1970 & Synopsis of Anatomy \\
\hline Ger et al. & 1996 & $\begin{array}{l}\text { Essentials of Clinical } \\
\text { Anatomy }\end{array}$ \\
\hline $\begin{array}{l}\text { Gosling } \\
\text { et al. }\end{array}$ & 1985 & Atlas of Human Anatomy \\
\hline Lumley & 2002 & Surface Anatomy \\
\hline $\begin{array}{l}\text { Lumley } \\
\text { et al. }\end{array}$ & 1995 & Essential Anatomy \\
\hline Martin & 1985 & $\begin{array}{l}\text { Introduction to Human } \\
\text { Anatomy }\end{array}$ \\
\hline $\begin{array}{l}\text { McMinn } \\
\text { et al. }\end{array}$ & 1984 & $\begin{array}{l}\text { Color Atlas of Applied } \\
\text { Anatomy }\end{array}$ \\
\hline $\begin{array}{c}\text { Putz and } \\
\text { Pabst }\end{array}$ & 2000 & $\begin{array}{l}\text { Sobotta's Atlas of } \\
\text { Human Anatomy }\end{array}$ \\
\hline Rohen et al. & 2002 & Color Atlas of Anatomy \\
\hline Tobin & 1973 & Basic Human Anatomy \\
\hline
\end{tabular}

beginning student, who may be searching for logic within the nomenclature. A true morass of nomenclature was found in one book in the current review (Lumley, 2002). The cutaneous innervation of the lower limb contained the "femoral branch of the genitofemoral," the "lateral cutaneous nerve of the thigh," the "intermediate femoral cutaneous," "medial cutaneous nerve of the thigh," "lateral cutaneous nerve of the leg," "musculocutaneous," "sural," and "anterior tibial” (Lumley, 2002). 


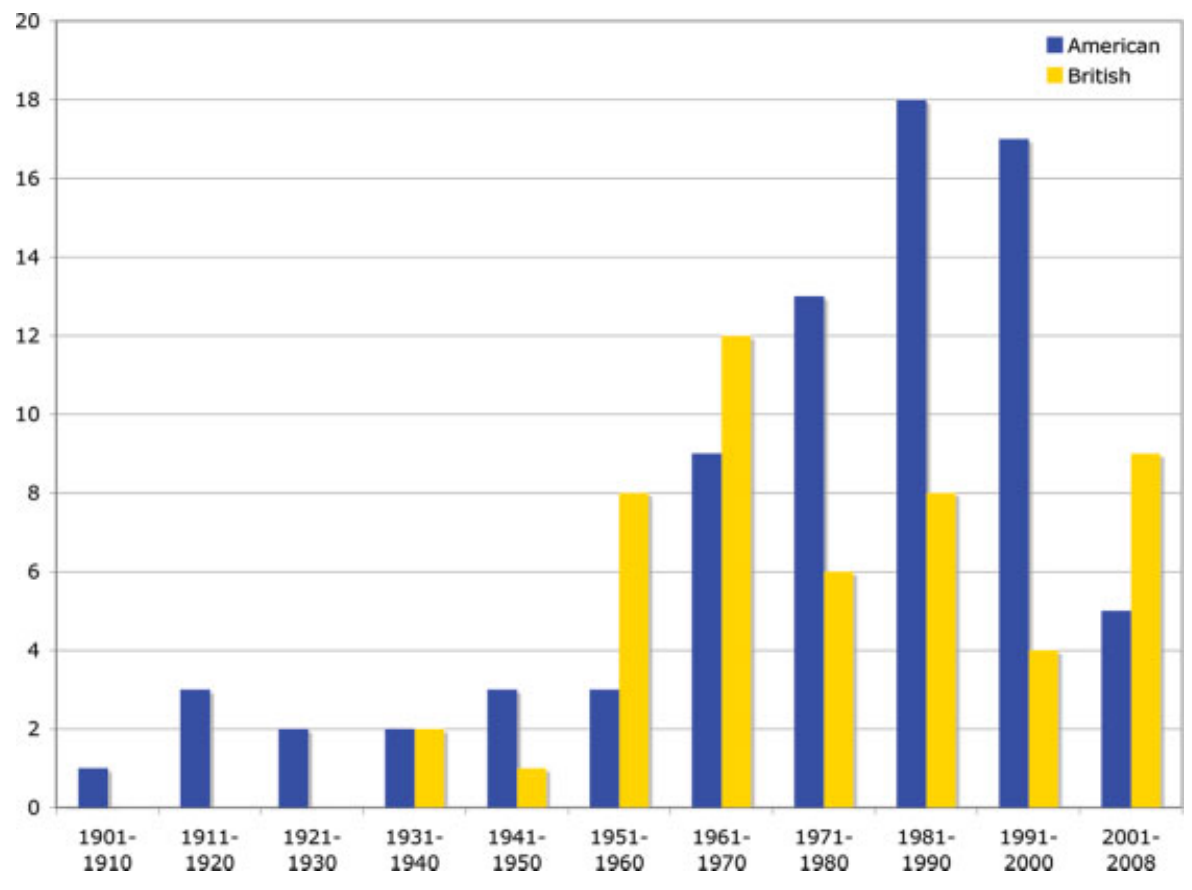

\section{Figure 1.}

Numbers of anatomy textbooks and atlases using American and British nomenclature systems, by decade.

If the British system of Latin equivalents for cutaneous nerves is consistently applied to all structures of the body, the system of anatomical nomenclature fails, and this failure might lead to catastrophic consequences. For example, "A. femoris" becomes "thigh artery," "N. femoris" becomes "thigh nerve," and anatomical terminology becomes common terms at the expense of precise communication of structures. Imagine the physician working on a gunshot wound to the thigh in an emergency room. Since there are at least several arteries in the thigh, imagine how rapid and accurate communication regarding ligating "A thigh artery" or "THE thigh artery" would be affected. Anatomical terminology exists to provide a precise, efficient, and intelligible description of human structure. It is our duty as anatomy educators to make anatomical nomenclature precise, logical, and consistent, so that our students can acquire their foundation in anatomy with the least amount of difficulty.

In his book, The Tipping Point, Malcolm Gladwell discusses the fact that before the battles of Lexington and Concord, two riders warned of the British coming: William Dawes and Paul Revere (Gladwell, 2002). One of these two men had little effect in mobilizing the militia and is not remembered, while the other succeeded in spreading his message. We hope that this article can be more like the later. We are not advocating a nomenclatural revolutionary war, but we hope that consistency and logic will tip the Battle of the Cutaneous Nerves.

\section{NOTES ON CONTRIBUTORS}

THOMAS R. GEST, Ph.D., is an associate professor of Anatomical Sciences and Medical Education at the University of Michigan Medical School, Ann Arbor, Michigan. He directs first year medical gross anatomy and fourth year electives in surgical anatomy.

WILLIAM E. BURKEL, Ph.D., is a professor emeritus of Anatomical Sciences at the University of Michigan Medical School, Ann Arbor, Michigan. He is enjoying his retirement.

GERALD W. CORTRIGHT, Ph.D., is a lecturer in Anatomical Sciences at the University of Michigan Medical School, Ann Arbor, Michigan. He directs first year dental gross anatomy and teaches first year medical gross anatomy.

\section{LITERATURE CITED}

Abrahams PH, Marks SC, Hutchings RT. 2003. McMinn's Colour Atlas of Human Anatomy. 5th Ed. New York, NY: Mosby. 378 p.

Agur AMR. 1991. Grant's Atlas of Anatomy. 9th Ed. Baltimore, MD: Williams \& Wilkins. 650 p.

Agur AMR, Dalley AF. 2005. Grant's Atlas of Anatomy. 11th Ed. Philadelphia, PA: Lippincott, Williams \& Wilkins. 848 p.

Aitken JT, Causey G, Joseph J, Young JZ. 1956. A Manual of Human Anatomy III. 1st Ed. Edinburgh, Scotland: E. \& S. Livingstone. 135 p.

Akesson EJ, Loeb JA, Wilson-Pauwels L. 1990. Thompson's Core Textbook of Anatomy. 2nd Ed. Philadelphia, PA: Lippincott, Williams \& Wilkins. 586 p.

Anderson JE. 1978. Grant's Atlas of Anatomy. 7th Ed. Baltimore, MD: Williams \& Wilkins. 600 p.

Anderson JE. 1983. Grant's Atlas of Anatomy. 8th Ed. Baltimore, MD: Williams \& Wilkins. 700 p.

Anson BJ. 1950. An Atlas of Human Anatomy. 1st Ed. Philadelphia, PA: W.B. Saunders. $518 \mathrm{p}$.

Anson BJ. (ed.) 1966. Morris' Human Anatomy. 12th Ed. New York, NY: McGraw-Hill. 1623 p.

Anson BJ, Maddock WG. (eds.) 1952. Callander's Surgical Anatomy. 3rd Ed. Philadelphia, PA: W.B. Saunders. 1074 p.

Arnold M. 1968. Reconstructive Anatomy. 1st Ed. Philadelphia, PA: W.B. Saunders. $529 \mathrm{p}$.

Backhouse KM, Hutchings RT. 1998. Clinical Surface Anatomy. 2nd Ed. London, UK: Mosby, Wolfe. 138 p. 
Basmajian JV. 1976. Primary Anatomy. 7th Ed. Baltimore, MD: Williams \& Wilkins. 405 p.

Basmajian JV, Slonecker CE. 1989. Grant's Method of Anatomy. 11th Ed. Baltimore, MD: Williams \& Wilkins. 615 p.

Beck EW. 1982. Mosby's Atlas of Functional Human Anatomy. 1st Ed. St. Louis, MO: Mosby. 310 p.

Bell C. 1834. The Anatomy and Physiology of the Human Body. Vol. 1. 6th American Ed. New York, NY: Collins and Co. 613 p.

Boyd JD, LeGros Clark WE, Hamilton WJ, Yoffey JM, Zuckerman S, Appleton AB. 1956. Textbook of Human Anatomy. 1st Ed. New York, NY: St. Martins Press. $1022 \mathrm{p}$.

Brantigan O. 1963. Clinical Anatomy. 1st Ed. New York, NY: McGraw-Hill. $421 \mathrm{p}$.

Brash JC. 1957. Cunningham's Manual of Practical Anatomy. 12th Ed. London, UK: Oxford University Press.

Callander CL. 1933. Surgical Anatomy. 1st Ed. Philadelphia, PA: W.B. Saunders. $1115 \mathrm{p}$.

Callander CL. 1939. Surgical Anatomy. 2nd Ed. Philadelphia: W.B. Saunders. 858 p.

Callas G. 1994. Manual for Human Dissection. 1st Ed. Norwalk, CT: Appleton and Lange. 203 p.

Cartmill M, Hylander WL, Shafland J. 1987. Human Structure. 1st Ed. Cambridge, MA: Harvard University Press. 448 p.

Christensen JB, Telford IR. 1966. Synopsis of Gross Anatomy. 1st Ed. New York: Harper and Row. 248 p.

Chung KW. 1991. Gross Anatomy. 2nd Ed. Media, PA: Harwal Pub. 377 p.

Clemente CD. 1975. Anatomy: A Regional Atlas of the Human Body. 1st Ed. Philadelphia, PA: Lea and Febiger. 320 p.

Clemente CD. 1985. Gray's Anatomy. 13th American Ed. Philadelphia, PA: Lea and Febiger. 1676 p.

Clemente CD. 1997. Anatomy: A Regional Atlas of the Human Body. 4th Ed. Baltimore, MD: Williams \& Wilkins. 576 p.

Colborn GL, Skandalakis JE. 1993. Clinical Gross Anatomy. 1st Ed. Pearl River, NY: Parthenon Pub. 581 p.

Crafts RC. 1966. A Textbook of Human Anatomy. 1st Ed. New York, NY: Ronald Press. 752 p.

Crafts RC. 1985. A Textbook of Human Anatomy. 3rd Ed. New York, NY: Wiley. 906 p.

Crouch JE. 1972. Functional Human Anatomy. 2nd Ed. Philadelphia, PA: Lea and Febiger. 649 p.

Dawson HL. 1996. Basic Human Anatomy. 1st Ed. New York, NY: Appleton, Century, Crofts. 332 p.

DiDio LJA. 1970. Synopsis of Anatomy. 1st Ed. St. Louis, MO: Mosby. 530 p. Drake RL, Vogl W, Mitchell AWM. 2005. Gray's Anatomy for Students. Philadelphia, PA: Elsevier. 1058 p.

Ellis H. 1960. Clinical Anatomy. 1st Ed. Oxford, UK: Blackwell. 369 p.

Ellis H. 1966. Clinical Anatomy. 3rd Ed. Oxford, UK: Blackwell. 381 p.

Ellis H, Logan BM, Dixon AK. 1999. Human Sectional Anatomy. 2nd Ed. London, UK: Hodder Arnold. 245 p.

FCAT. 1998. Federative Committee on Anatomical Terminology, Terminologia Anatomica: International Anatomical Terminology. 1st Ed. Stuttgart, Germany: Thieme. 292 p.

Francis C. 1959. Introduction to Human Anatomy. 3rd Ed St. Louis, MO: Mosby. 548 p.

Frick H, Leonhardt H, Starck D. (trans. Meyer). 1991. Human Anatomy. 1st Ed. New York, NY: Thieme Medical Publishers. 1396 p.

Gardner ED, O'Rahilly RO, Müller F. 1986. Anatomy: A Regional Study of Human Structure. 5th Ed. Philadelphia, PA: W. B. Saunders. 809 p.

Gardner WD, Osburn WA. 1973. Structure of the Human Body. 2nd Ed. Philadelphia, PA: Saunders. 516 p.

Gardner WD, Osburn WA. 1978. Anatomy of the Human Body. 3rd Ed. Philadelphia, PA: Saunders. 563 p.

Ger R, Abrahams P, Olson TR. 1996. Essentials of Clinical Anatomy. 1st Ed. New York, NY: Parthenon Publishing Group. 544 p.

Gest TR, Schlesinger J. 1994. Medcharts: Anatomy. 1st Ed. New York, NY: ILOC. 309 p.

Gladwell M. 2002. The Tipping Point. 1st Ed. New York, NY: Little, Brown and Company. 304 p.

Gosling JA, Harris PF, Humpherson JR, Whitmore I, Willan PLT. 1985. Atlas of Human Anatomy. 1st Ed. London, UK: Gower Medical. 310 p.

Gosling JA, Harris PF, Whitmore I, Willan PLT. 2002. Human Anatomy Color Atlas and Text. 4th Ed. Edinburgh, Scotland: Mosby. 377 p.

Goss CM. 1954. Gray's Anatomy of the Human Body. 26th Ed. Philadelphia, PA: Lea and Febiger. 1480 p.

Goss CM. 1959. Gray's Anatomy of the Human Body. 27th Ed. Philadelphia, PA: Lea and Febiger. 1458 p.

Goss CM. 1966. Gray's Anatomy of the Human Body. 28th Ed. Philadelphia, PA: Lea and Febiger. 1448 p.
Gottlieb MI. 1964. Anatomy Review. 2nd Ed. Flushing, NY: Medical Examination Publishing Company. 210 p.

Grant JC. 1958. A Method of Anatomy. 6th Ed. Baltimore, MD: Williams \& Wilkins. 879 p.

Grant JC. 1962. An Atlas of Anatomy. 5th Ed. Baltimore, MD: Williams \& Wilkins.

Grant JC, Basmajian JV. 1965. Grant's Method of Anatomy. 7th Ed. Baltimore, MD: Williams \& Wilkins. 777 p.

Gray H. 1858. Anatomy, Descriptive and Surgical. 1st Ed. Philadelphia, PA: Blanchard and Lea. 754 p.

Gray H, Goss CM. (eds.) 1948. Anatomy of the Human Body. 25th Ed. Philadelphia, PA: Lea and Febiger. 1478 p.

Green JH, Silver PHS. 1981. An Introduction to Human Anatomy. 1st Ed. New York, NY: Oxford University Press. 424 p.

Hall-Craggs ECB. 1990. Anatomy as a Basis for Clinical Medicine. 2nd Ed. Baltimore, MD: Williams \& Wilkins. 593 p.

Hamilton WJ. (ed.) 1976. Textbook of Human Anatomy. 2nd Ed. London, UK: Macmillan. 753 p.

Hansen JT. 1998. Essential Anatomy Dissector. 1st Ed. Baltimore, MD: Williams \& Wilkins. 191 p.

Healey JE. 1969. A Synopsis of Clinical Anatomy. 1st Ed. Philadelphia, PA: Saunders. 324 p.

His W. 1895. Die anatomische Nomenclatur. (Basel) Nomina Anatomica. 1st Ed. Arch Anat Phys Anat Abth Suppl. Leipzig, Germany: Veit. 180 p.

Hollinshead WH. 1969. Anatomy for Surgeons. 2nd Ed. New York, NY: Hoeber Medical. 619 p.

Hollinshead WH. 1974. Textbook of Anatomy. 3rd Ed. New York, NY: Harper and Row. 985 p.

Hollinshead WH, Rosse C. 1985. Textbook of Anatomy. 4th Ed. Philadelphia: Harper and Row. 1041 p.

Huber GC. (ed.) 1930. Piersol's Human Anatomy. 9th Ed. Philadelphia, PA: Lippincott. 2104 p.

IANC. 1983. International Anatomical Nomenclature Committee, Nomina Anatomica: A Revision by the International Anatomical Nomenclature Committee Approved by the Eleventh International Congress of Anatomists in Mexico City, 1980. 5th Ed. Baltimore, MD: Williams \& Wilkins. 86 p.

Jackson CM. 1914. Morris's Human Anatomy. 5th Ed. Philadelphia, PA: P. Blakiston's Son.

Jacobs JJ. (ed.) 1989. Shearer's Manual of Human Dissection. 7th Ed. New York, NY: McGraw-Hill. 288 p.

Jacob S. 2002. Atlas of Human Anatomy. 1st Ed. New York, NY: Churchill Livingstone. $252 \mathrm{p}$.

Jamieson EB. 1939. Illustrations of Regional Anatomy. 2nd Ed. Edinburgh, Scotland: E. \& S. Livingstone. Section VI: 42 p. and Section VII: 52 p.

Jamieson EB. 1950. A Companion to Manuals of Practical Anatomy. 7th Ed. New York, NY: Oxford University Press. 736 p.

Kahle W, Leonhardt H, Platzer W. 1992. Color Atlas and Textbook of Human Anatomy. Locomotor System. Vol. 1. 4th Ed. Stuttgart, Germany: G. Thieme Verlag. 435 p.

Kanagasuntheram R, Sivanandasingham P, Krishnamurti A. 1987 Anatomy: Regional, Functional and Clinical. 1st Ed. Singapore: P G Publishers. $649 \mathrm{p}$.

Lachman E. 1965. Case Studies in Anatomy. 1st Ed. New York, NY: Oxford University Press. 238 p.

Langebartel DA. 1977. The Anatomical Primer: An Embryological Explanation of Human Gross Morphology. 1st Ed. Baltimore, MD: University Park Press. $510 \mathrm{p}$.

Langman J, Woerdeman MW. 1978. Atlas of Medical Anatomy. 1st Ed. Philadelphia, PA: Saunders. 523 p.

Last RJ. 1955. Aids to Anatomy. 11th Ed. London, UK: Bailliére, Tindall and Cox. 379 p.

Leeson CR, Leeson TS. 1972. Human Structure: A Companion to Anatomical Studies. 1st Ed. Philadelphia, PA: Saunders. 383 p.

Leonard RJ. 1995. Human Gross Anatomy. 1st Ed. New York, NY: Oxford University Press. 437 p.

Lewis WH. 1918. Gray's Anatomy of the Human Body. 20th Ed. Philadelphia, PA: Lea and Febiger. 1396 p.

Lewis WH. 1936. Gray's Anatomy of the Human Body. 23rd Ed. Philadelphia, PA: Lea and Febiger. 1381 p.

Lewis WH. 1942. Gray's Anatomy of the Human Body. 24th Ed. Philadelphia, PA: Lea and Febiger. 1428 p.

Lockhart RD, Hamilton GF, Fyfe FW. 1959. Anatomy of the Human Body. 1st Ed. Philadelphia, PA: Lippincott. 697 p.

Lopez-Antunez L, Gasparo LA. 1971. Atlas of Human Anatomy. 1st Ed. Philadelphia, PA: Saunders. 366 p.

Lumley JSP. 2002. Surface Anatomy. 3rd Ed. Edinburgh, Scotland: Churchill Livingstone. $135 \mathrm{p}$. 
Lumley JSP, Craven JL, Aiken JT. 1995. Essential Anatomy. 5th Ed. Edinburgh, Scotland: Churchill Livingstone. 592 p.

Martin AH. 1985. Introduction to Human Anatomy. 1st Ed. New York, NY: Thieme-Stratton. $572 \mathrm{p}$.

McMinn RMH, Hutchings RT. 1977. Color Atlas of Human Anatomy. 1st Ed. Chicago, IL: Year Book Medical Publishers. $352 \mathrm{p}$.

McMinn RMH, Hutchings RT, Logan BM. 1984. Color Atlas of Applied Anatomy. 1st Ed. Chicago, IL: Year Book Medical Publishers. 214 p.

McMinn RMH, Hutchings RT. 1988. Color Atlas of Human Anatomy. 2nd Ed. Chicago, IL: Year Book Medical Publishers. 358 p.

Melloni JL, Dox I, Melloni HP, Melloni BJ. 1988. Melloni's Illustrated Review of Human Anatomy. 1st Ed. Philadelphia, PA: Lippincott. 268 p.

Mitchell GA, Patterson EL. 1967. Basic Anatomy. 2nd Ed. Edinburgh, Scotland: E. \& S. Livingstone. 623 p.

Mizeres NJ. 1981. Human Anatomy: A Synoptic Approach. 1st Ed. New York, NY: Elsevier. 341 p.

Moore KL. 1992. Clinically Oriented Anatomy. 3rd Ed. Baltimore, MD: Williams \& Wilkins. $917 \mathrm{p}$

Moore KL, Dalley AF. 2006. Clinically Oriented Anatomy. 5th Ed. Philadelphia, PA: Lippincott, Williams \& Wilkins. 1209 p.

Morton DA, Peterson KD, Albertine KH. 2004. 1st Ed. Dissection Guide for Human Anatomy. Philadelphia, PA: Churchill Livingstone. 466 p.

Netter FH. 1987. The Ciba Collection of Medical Illustrations. Vol. 13. 1st Ed. Summit, NJ: CIBA-GEIGY Corp.

Netter FH. 1989. Atlas of Human Anatomy. 1st Ed. Summit, NJ: CIBAGEIGY Corp. 550 p.

Netter FH, Dalley AF. 1997. Atlas of Human Anatomy. 2nd Ed. East Hanover, NJ: Novartis. 525 p.

Netter FH, Hansen JT. 2003. Atlas of Human Anatomy. 3rd Ed. Teterboro, NJ: Icon Learning Systems. 590 p.

Netter FH. 2006. Atlas of Human Anatomy. 4th Ed. Philadelphia, PA: Saunders. $640 \mathrm{p}$.

Olson TR, Pawlina W. 1996. A.D.A.M. Student Atlas of Anatomy. 1st Ed. Baltimore, MD: Williams \& Wilkins. 492 p.

O’Rahilly RO. Müller F. 1983. Basic Human Anatomy: A Regional Study of Human Structure. 1st Ed. Philadelphia, PA: Saunders. 566 p.

Pansky B, House EL. 1975. Review of Gross Anatomy. 3rd Ed. New York, NY: Macmillan. 508 p.

Pauchet V, Dupret S. 1937. Pocket Atlas of Anatomy. 3rd Ed. London, UK: Oxford University Press. 368 p.

Pernkopf E, Ferner H, Monsen H. (eds.) 1964. Atlas of Topographical and Applied Human Anatomy. 2nd Ed. München, Germany: Urban \& Schwarzenberg. $777 \mathrm{p}$.

Pernkopf E. 1980. Atlas of Topographical and Applied Human Anatomy. 3rd Ed. Wien, Austria: Urban \& Schwarzenberg. 778 p.

Philo R, Bosner MS, LeMaistre A, Linner JG, Venger BH. 1985. Guide to Human Anatomy. 1st Ed. Philadelphia, PA: Saunders. 335 p.

Pick TP, Howden R. (eds.) 1901. Anatomy, Descriptive and Surgical. 15th Ed. London, UK: Longmans, Green. 1096 p.

Piersol GA. (ed.) 1923. Human Anatomy. 8th Ed. Philadelphia, PA: Lippincott. $2104 \mathrm{p}$.

Putz R, Pabst R. 2000. Sobotta's Atlas of Human Anatomy. 13th Ed. Philadelphia, PA: Lippincott Williams \& Wilkins. 833 p.

Quiring DP, Warfel JH. 1960. The Extremities. 2nd Ed. Philadelphia, PA: Lea and Febiger. 120 p.

Rarey KE, Romrell LJ, Pawlina W, Rosenberg JJ. 1994. Human Anatomy: Manual of Human Dissection. 1st Ed. Gainesville, FL: Gold Standard Multimedia. $258 \mathrm{p}$.

Rogers AW. 1992. Textbook of Anatomy. 1st Ed. Edinburgh, Scotland: Churchill Livingstone. $779 \mathrm{p}$

Rohen JW, Yokochi C, Lutjen-Drecoll E. 2002. Color Atlas of Anatomy. 5th Ed. Baltimore, MD: Lippincott Williams \& Wilkins. 500 p.

Romanes GJ. 1964. Cunningham's Textbook of Anatomy. 10th Ed. New York, NY: Oxford University Press. 1014 p.

Romanes GJ. 1986. Cunningham's Manual of Practical Anatomy. Upper and Lower Limbs. Vol. 1. 15th Ed. New York, NY: Oxford University Press. 240 p.

Rosse C, Gaddum-Rosse P. 1997. Hollinshead's Textbook of Anatomy. 5th Ed. Philadelphia, PA: Lippincott-Raven Publishers. 902 p.

Sahana SN. 1962. Human Anatomy. 1st Ed. Calcutta, India: Central Book Agency. 1244 p.
Sauerland EK. 1984. Grant's Dissector. 9th Ed. Baltimore, MD: Williams \& Wilkins. $187 \mathrm{p}$.

Schade JP. 1970. Introduction to Functional Human Anatomy. 1st Ed. Philadelphia, PA: Saunders. 189 p.

Schaeffer JP. (ed.) 1942. Morris' Human Anatomy. 10th Ed. Philadelphia, PA: Blakiston. $1641 \mathrm{p}$.

Sinclair D. 1961. An Introduction to Functional Anatomy. 2nd Ed. Oxford, UK: Blackwell. $447 \mathrm{p}$.

Slaby FJ, McCune SK, Summers RW. 1994. Gross Anatomy in the Practice of Medicine. 1st Ed. Philadelphia, PA: Lea and Febiger. 699 p.

Snell RS. 1978. Atlas of Clinical Anatomy. 1st Ed. Boston, MA: Little, Brown. $530 \mathrm{p}$.

Snell RS. 1995. Clinical Anatomy for Medical Students. 5th Ed. Boston, MA: Little, Brown. 898 p.

Sobotta J, McMurrich JP. 1906. Atlas and Text-Book of Human Anatomy. Vol. 3. 1st Ed. Philadelphia, PA: W.B. Saunders. 342 p.

Sobotta J, McMurrich JP. 1928. Atlas of Human Anatomy. 2nd Ed. Philadelphia, PA: W.B. Saunders. 785 p.

Sobotta J, McMurrich JP. 1936. Atlas of Human Anatomy. Vol. 3. 4th Ed. New York, NY: Stechert. 350 p.

Spalteholz W. 1900. Hand-Atlas of Human Anatomy. 3rd Ed. Leipzig, Germany: S. Hirzel. $902 \mathrm{p}$.

Spalteholz W, Spanner R. 1967. Atlas of Human Anatomy. 16th Ed. Philadelphia, PA: F.A. Davis Co. 904 p.

Spence AP. 1986. Basic Human Anatomy. 2nd Ed. Menlo Park, CA: Benjamin/ Cummings Pub. Co. 688 p.

Srebnik HH. 2002. Concepts in Anatomy. 1st Ed. Boston, MA: Kluwer Academic Publishers. 239 p.

Standring S, Ellis H, Healy JC, Johnson D, Williams A. 2005. Gray's Anatomy. 39th Ed. Edinburgh, Scotland; New York, NY: Elsevier Churchill Livingstone. $1627 \mathrm{p}$.

Stern JT. 1988. Essentials of Gross Anatomy. 1st Ed. Philadelphia, PA: Davis. $795 \mathrm{p}$.

Tank PW. 2008. Grant's Dissector. 14th Ed. Philadelphia, PA: Lippincott, Williams \& Wilkins. 288 p.

Tank PW, Gest TR. 2008. Lippincott Williams \& Wilkins Atlas of Anatomy. 1st Ed. Philadelphia, PA: Lippincott, Williams \& Wilkins. 432 p.

Thiel W. 1997. Photographic Atlas of Practical Anatomy. 1st Ed. Berlin, Germany: Springer. $417 \mathrm{p}$.

Thompson JS. 1977. Core Textbook of Anatomy. 1st Ed. Philadelphia, PA: Lippincott. $431 \mathrm{p}$.

Thompson JC. (ed.) 2002. Netter's Concise Atlas of Orthopaedic Anatomy. 1st Ed. Teterboro, NJ: Icon Learning Systems. 320 p.

Thorek P. 1962. Anatomy in Surgery. 2nd Ed. Philadelphia, PA: Lippincott. $904 \mathrm{p}$.

Tobin CE. 1973. Basic Human Anatomy. 1st Ed. New York, NY: McGrawHill. 342 p.

Van De Graaff KM. 2002. Human Anatomy. 6th Ed. Boston, MA: McGrawHill Higher Education. $840 \mathrm{p}$.

Vidić B. 1984. Manual of Dissection. 1st Ed. St. Louis, MO: C.V. Mosby. 118 p.

Vidić B, Suarez FR. 1984. Photographic Atlas of the Human Body. 1st Ed. St. Louis, MO: C.V. Mosby. 464 p.

Wilson DB, Wilson WJ. 1978. Human Anatomy. 1st Ed. Baltimore, MD: Williams \& Wilkins. 454 p.

Wilson E, Gobrecht WH. (eds.) 1858. A system of human anatomy. 7th Ed. Philadelphia, PA: Blanchard and Lea. 616 p.

Wilson JL. 1988. Dissection Manual. 2nd Ed. New York, NY: Igaku-Shoin Medical Publishers. 244 p.

Wischnitzer S. 1972. Outline of Human Anatomy. 1st Ed. Springfield, IL: Thomas. 392 p.

Wolf-Heidegger G. 1962. Atlas of Systematic Human Anatomy. 1st Ed. New York, NY: Hafner Pub. Co. 635 p.

Woodburne RT, Burkel WE. 1994. Essentials of Human Anatomy. 9th Ed. New York, NY: Oxford University Press. 687 p.

Zuckerman S. 1961. A New System of Anatomy. 1st Ed. London, UK: Oxford University Press. 579 p.

Zuidema GD. 1980. The Johns Hopkins Atlas of Human Anatomy. 2nd Ed. Baltimore, MD: Johns Hopkins University Press. 115 p. 